\title{
Locoregional recurrence patterns in women with breast cancer who have not undergone post-mastectomy radiotherapy
}

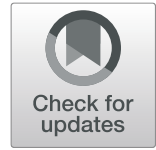

\author{
Xuran Zhao ${ }^{\dagger}$, Yu Tang ${ }^{\dagger}$, Shulian Wang ${ }^{*}$, Yong Yang, Hui Fang, Jianyang Wang, Hao Jing, Jianghu Zhang, \\ Guangyi Sun, Siye Chen, Jing Jin, Yongwen Song, Yueping Liu, Bo Chen, Shunan Qi, Ning Li, Yuan Tang, \\ Ningning Lu, Hua Ren and Yexiong Li ${ }^{*}$
}

\begin{abstract}
Background: To analyze the patterns of locoregional recurrence in breast cancer patients after mastectomy. Methods: The retrospective study included 7073 women with breast cancer without post-mastectomy radiotherapy: 4604 (65.1\%) had pT1-2 N0 disease (low risk); 2042 (28.9\%), pT1-2 N1 (intermediate risk); and 427 (6.0\%), pT3-4 and/or pN2-3, or pT1-2 N1 after neoadjuvant chemotherapy (high risk). The distribution of cumulative locoregional recurrence was analyzed. The local recurrence and regional recurrence rates were estimated by the Kaplan-Meier method, and differences were compared with the log-rank test. Multivariate analysis was performed using Cox logistic regression analysis.

Results: In the median follow-up of 63.0 months, 469 patients had locoregional recurrence: chest wall recurrence in 238 (50.7\%) cases, supraclavicular/infraclavicular nodes in 236 (50.3\%) cases, axilla in 92 (19.6\%), and internal mammary nodes in 50 (10.7\%) cases. The 5-year local recurrence and regional recurrence rates were 2.5 and $4.4 \%$, respectively. Subgroup analysis of the three risk groups and five molecular subtypes (luminal A, luminal B-Her2 negative, luminal B-Her2 positive, Her2-enriched, and triple negative) also showed that the chest wall and supraclavicular/infraclavicular nodes were the most common recurrence sites. Age, tumor location, T stage, N stage, and hormone receptor status were independent prognostic factors for both local recurrence and regional recurrence $(p<0.05)$.

Conclusions: The chest wall and supraclavicular/infraclavicular nodes are common sites of locoregional recurrence in breast cancer, irrespective of disease stage or molecular subtype, and the prognostic factors for local recurrence and regional recurrence are similar. Therefore, chest wall and supraclavicular/infraclavicular nodes irradiation should always be considered in post-mastectomy radiotherapy.
\end{abstract}

Keywords: Breast neoplasm, Mastectomy, Locoregional recurrence, Risk factors

\footnotetext{
*Correspondence: wsl20040118@yahoo.com; yexiong@yahoo.com

${ }^{+}$Xuran Zhao and Yu Tang contributed equally as co-first author.

Department of Radiation Oncology, National Cancer Center/National Clinical

Research Center for Cancer/Cancer Hospital, Chinese Academy of Medical

Sciences and Peking Union Medical College, 17 Panjiayuannanli, Chaoyang

District, Beijing 100021, China
}

C C The Author(s). 2020 Open Access This article is licensed under a Creative Commons Attribution 4.0 International License, which permits use, sharing, adaptation, distribution and reproduction in any medium or format, as long as you give appropriate credit to the original author(s) and the source, provide a link to the Creative Commons licence, and indicate if changes were made. The images or other third party material in this article are included in the article's Creative Commons licence, unless indicated otherwise in a credit line to the material. If material is not included in the article's Creative Commons licence and your intended use is not permitted by statutory regulation or exceeds the permitted use, you will need to obtain permission directly from the copyright holder. To view a copy of this licence, visit http://creativecommons.org/licenses/by/4.0/ The Creative Commons Public Domain Dedication waiver (http://creativecommons.org/publicdomain/zero/1.0/) applies to the data made available in this article, unless otherwise stated in a credit line to the data. 


\section{Background}

Postmastectomy radiotherapy (PMRT) is an important strategy for the locoregional management of breast cancer, as it can reduce the risk of locoregional recurrence (LRR) and decrease breast cancer mortality [1]. Previous randomized studies have shown that chest wall and comprehensive regional nodal irradiation result in a decrease in LRR and an improvement in overall survival in patients with node-positive or T3-4 disease [2-4]. However, the recurrence patterns have changed with the advances in diagnostic technologies and therapeutic approaches, and as a result, the optimal radiation target volume is now under debate. Some studies have shown that the risk of regional nodal recurrence in patients with 1-3 positive nodes is relatively low [5-9], but it is unclear whether comprehensive locoregional radiotherapy is essential for improving survival, or whether delivering radiation to a more limited target volume may achieve a comparable outcome. In addition, several studies have examined whether high-risk patients with T1-2N0 disease are suitable for PMRT, but the optimal radiation target volume is unclear [10-14].

Understanding the local and regional recurrence patterns in more recent cohorts of patients could help in making decisions about radiotherapy, such as the ideal target volume. Although some studies have investigated the patterns of LRR after mastectomy, the results were limited either by their small sample size, or by the type of population and time period studied. Further, there is little information about LRR patterns after modern systemic therapy, such as standard hormone therapy and anthracycline- or taxane-based chemotherapy, although there is evidence that the modern therapies provide superior outcome to traditional regimens for nodepositive patients [15-17]. Additionally, different molecular subtypes are associated with different prognoses, so treatment regimens are typically personalized to the needs of individual patients [18]. However, the association of molecular subtypes with LRR is unclear. In this study, we seek to fill in all these information gaps by analyzing the patterns of LRR in a large series of patients with breast cancer who underwent mastectomy along with modern treatment regimens, and by investigating whether locoregional failure was related to disease stage or molecular subtypes.

\section{Methods}

This study was approved by our Institutional Review Board (approval number 15-057/984). Patients with pathologically confirmed breast cancer who underwent mastectomy between January 1999 and June 2014 and met the following criteria were eligible: $18-75$ years of age, no PMRT, no evidence of distant dissemination or supraclavicular internal mammary nodal metastasis at diagnosis, no prior or concurrent malignancy, and $\geq 6$ months of follow-up after mastectomy (Fig. 1).

The molecular subtypes were constructed according to the ER, PR, and HER2 status, and the tumors were graded into five categories: (1) luminal A: $\mathrm{ER}+$ or $\mathrm{PR}+$, HER2-, and grade 1 or 2; (2) Luminal B-Her2 negative: ER+ or PR+, HER2-, and grade 3; (3) Luminal B-Her2 positive: ER+ or $\mathrm{PR}+$, and HER2+; (4) Her2-enriched: ER-, PR- and HER2+; and (5) triple-negative breast cancer (TNBC): ER-, PR-, and HER2-. Tumor grade was used to construct the molecular subtypes, because the Ki-67 index was only available for 1436 (20.3\%) patients.

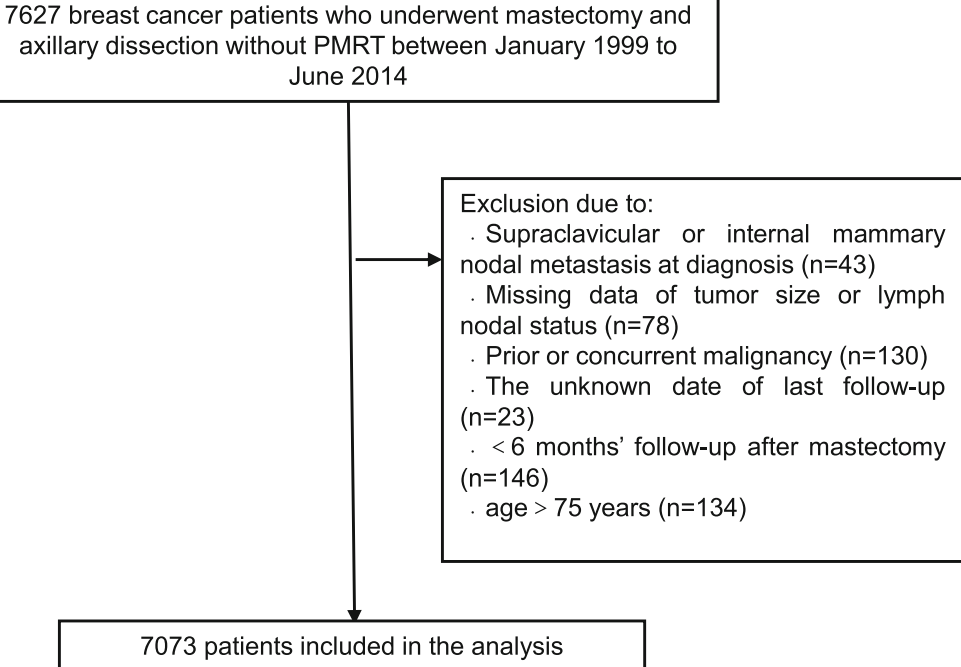

7073 patients included in the analysis

Fig. 1 Flow diagram showing patient selection 
Follow-up data were obtained from hospital records or from correspondence directly with the patient or their family. All recurrences were confirmed by pathologic or radiographic evidence. LRR included local recurrence (LR) and/or regional recurrence (RR). LR was defined as recurrence in the ipsilateral chest wall, and $R R$ was defined as recurrence involving the ipsilateral axillary, supraclavicular/infraclavicular, or internal mammary lymph nodes that was detected during follow-up, regardless of whether distant metastasis occurred earlier, later, or simultaneously. Recurrence at any other site was considered as distant metastasis. The cumulative LR, RR, and LRR rates were estimated by the Kaplan-Meier method, and the differences were compared with the log-rank test. The recurrence rates were calculated from the date of surgery. Multivariate analysis was performed using Cox logistic regression analysis. Significant variables (as indicated by a significance value of $P<0.05$ ) identified from the univariate analysis were included in the Cox regression model. All $P$ values were two-tailed, and a value of less than 0.05 was considered to be indicate significance. All statistical analyses were carried out using the SPSS Package for Windows, version 24.0 (SPSS Inc., Chicago, IL, USA).

\section{Results}

The present study cohort comprised 7073 patients. The patient, tumor, and treatment characteristics are summarized in Table 1 . The median age was 50 years (range, 19-75 years). Axillary lymph node dissection was performed in 6734 (95.2\%) patients, and 339 (4.8\%) patients with pN0 disease underwent sentinel node biopsy alone. The median number of axillary lymph nodes dissected was 19 (range, 1-63). According to the seventh edition of the American Joint Committee on Cancer (AJCC) Staging System for breast cancer, patients were divided into three groups based on the tumor burden: the lowrisk group comprising 4604 (65.1\%) patients who had pT1-2 N0 disease, the intermediate-risk group comprising 2042 (28.9\%) patients who had pT1-2 N1 disease, and the high-risk group comprising 427 (6.0\%) patients (370 with pT3-4 or pN2-3 disease, and 57 with pT1-2 N1 disease after neoadjuvant chemotherapy). In 5330 cases, there was sufficient information for determination of the molecular subtype: 2397 (45.0\%), luminal A; 573 (10.8\%), luminal B-Her2 negative; 810 (15.2\%), luminal B-Her2 positive; 583 (10.9\%), Her2 enriched; and 967 (18.1\%), triple negative (TN).

Out of the 7073 patients in the cohort, 5373 (76.0\%) patients received neoadjuvant and/or adjuvant chemotherapy with a median of 6 cycles (range, 1-12). Among them, 5219 (97.1\%) received adjuvant chemotherapy and 154 (2.9\%) received neoadjuvant chemotherapy. The chemotherapy regimens included anthracycline and/or taxane in 4669 (86.9\%) patients, while other regimens were used in the remaining 704 (13.1\%) patients. Among the 5212 patients with hormone receptor-positive disease, $4400(84.4 \%)$ received adjuvant endocrine therapy. The median duration of endocrine therapy was 47 months (range, 1-160 months). In 1400 patients with Her2-positive disease, 321 (22.9\%) received trastuzumab therapy.

The median follow-up was 63.0 months (range, 6.0194.6 months), and 469 (6.6\%) patients developed LRR. Among them, 154 (32.8\%) had LR alone, 231 (49.3\%) had RR alone, and 84 (17.9\%) had both LR and RR (Fig. 2). Figure 3 showed the regional failure patterns for patients with RR. The 5-year cumulative incidence of LRR was $6.4 \%$. The distribution of LRR sites in the 469 patients was as follows: chest wall in 238 (50.7\%) cases, of which 170 (71.4\%) cases were pathologically confirmed; supraclavicular/infraclavicular nodes (SCN) in 236 (50.3\%) cases, of which 131 (55.5\%) cases were pathologically confirmed; axilla in $92(19.6 \%)$ cases, of which 45 (48.9\%) cases were pathologically confirmed; and internal mammary nodes (IMN) in 50 (10.7\%) cases, of which $20(40.0 \%)$ cases were pathologically confirmed. The distribution of LRR according to risk group and molecular subtype is shown in Table 2 and Table 3. Table 4 shows the distribution of LRR between Her2positive patients who did and did not receive targeted therapy (trastuzumab therapy), and between hormone receptor-positive patients who did and did not receive endocrine therapy.

A total of 238 patients (3.4\%) developed LR, and the 5year cumulative LR rate was $2.5 \%$. The median interval from surgery to LR was 52.4 months (range, 5.9-191.9 months). Further, 315 patients (4.5\%) developed RR, and the 5 -year cumulative RR rate was $4.4 \%$. The median interval from surgery to RR was 29.5 months (range, 0.6-149.4 months). The results of univariate analysis of the association of clinical variables with the risk of LR and $R R$ are shown in Table 1 . The significant variables identified in the univariate analysis were used for multivariate analysis, which showed that age $\leq 45$ years, location of the tumor in the inner quadrant, T3-4 stage disease, N1-3 stage disease, and hormone receptor negative were associated with increased risk of both LR and RR (Table 5). Since the SCN was the most common site of regional recurrence, we also investigated the prognostic factors of recurrence in the SCN, to assess whether statistical significance was still present for the variables analyzed. The univariate and multivariate analyses showed that the independent prognostic factors of recurrence in the SCN were the same as those of RR (supplementary Table 1). The LR and RR rates of patients with respect to different prognostic factors are displayed in Fig. 4. 
Table 1 Local and regional recurrence rates stratified by patient, tumor, and treatment characteristics

\begin{tabular}{|c|c|c|c|c|c|c|c|}
\hline Characteristic & No. of Patients (\%) & 5-year LR (\%) & $\mathrm{HR}(95 \% \mathrm{Cl})$ & $P$ & 5-year RR (\%) & $\mathrm{HR}(95 \% \mathrm{Cl})$ & $P$ \\
\hline Age & & & & 0.005 & & & $<0.001$ \\
\hline$>45$ years & $4864(68.8)$ & 2.2 & 1.000 & & 3.5 & 1.000 & \\
\hline$\leq 45$ years & $2209(31.2)$ & 3.1 & $1.450(1.118-1.879)$ & & 6.4 & $1.863(1.492-2.325)$ & \\
\hline Location & & & & $<0.001$ & & & $<0.001$ \\
\hline Other quadrants & $5211(73.7)$ & 1.9 & 1.000 & & 3.6 & 1.000 & \\
\hline Inner quadrant & $1686(23.8)$ & 3.6 & 1.729 (1.303-2.293) & & 6.0 & $1.663(1.305-2.120)$ & \\
\hline Unknown & $176(2.5)$ & & & & & & \\
\hline Pathologic type & & & & 0.210 & & & 0.058 \\
\hline Others & $893(12.6)$ & 1.7 & 1.000 & & 2.9 & 1.000 & \\
\hline Ductal & $6150(87.0)$ & 2.6 & 1.309 (0.858-1.996) & & 4.6 & $1.445(0.986-2.118)$ & \\
\hline Unknown & $30(0.4)$ & & & & & & \\
\hline T stage ${ }^{a}$ & & & & $<0.001$ & & & $<0.001$ \\
\hline $\mathrm{T} 1-2$ & $6967(98.5)$ & 2.2 & 1.000 & & 4.0 & 1.000 & \\
\hline T3-4 & $106(1.5)$ & 24.2 & 13.018 (8.813-19.231) & & 31.0 & $8.961(6.075-13.216)$ & \\
\hline$N$ stage $^{a}$ & & & & $<0.001$ & & & $<0.001$ \\
\hline NO & $4604(65.1)$ & 1.6 & 1.000 & & 2.5 & 1.000 & \\
\hline N1 & $2142(30.3)$ & 2.9 & $1.940(1.459-2.580)$ & & 5.9 & $2.922(2.271-3.761)$ & \\
\hline N2 & $173(2.4)$ & 9.1 & 5.549 (3.381-9.108) & & 12.2 & $6.124(3.825-9.804)$ & \\
\hline N3 & $154(2.2)$ & 17.6 & $14.513(9.488-22.199)$ & & 33.1 & $18.467(12.827-26.587)$ & \\
\hline No. of node dissected & & & & 0.035 & & & 0.007 \\
\hline$\geq 10$ & $6485(91.7)$ & 2.4 & 1.000 & & 4.3 & 1.000 & \\
\hline$<10$ & $588(8.3)$ & 4.9 & $1.598(1.029-2.481)$ & & 7.2 & $1.652(1.145-2.384)$ & \\
\hline Tumor grade & & & & 0.001 & & & 0.001 \\
\hline 1 & $409(5.8)$ & 0 & 1.000 & & 1.7 & 1.000 & \\
\hline 2 & $3678(52.0)$ & 2.1 & $4.748(1.171-19.244)$ & & 3.7 & $2.403(1.062-5.440)$ & \\
\hline 3 & $1713(24.2)$ & 3.2 & $7.004(1.715-28.607)$ & & 5.6 & $3.503(1.534-8.001)$ & \\
\hline Unknown & $1273(18.0)$ & & & & & & \\
\hline Lymphovascular invasion & & & & 0.252 & & & 0.062 \\
\hline No & $6668(94.3)$ & 2.4 & 1.000 & & 4.2 & 1.000 & \\
\hline Yes & $354(5.0)$ & 4.7 & $1.357(0.803-2.291)$ & & 6.9 & 1.507 (0.977-2.325) & \\
\hline Unknown & $51(0.7)$ & & & & & & \\
\hline Hormone receptor status & & & & $<0.001$ & & & $<0.001$ \\
\hline Positive & $5212(73.7)$ & 1.9 & 1.000 & & 3.3 & 1.000 & \\
\hline Negative & $1745(24.7)$ & 4.4 & $1.906(1.467-2.476)$ & & 7.8 & 1.990 (1.585-2.498) & \\
\hline Unknown & $116(1.6)$ & & & & & & \\
\hline HER2 status & & & & 0.196 & & & 0.001 \\
\hline Negative & $4536(64.1)$ & 2.2 & 1.000 & & 3.8 & 1.000 & \\
\hline Positive & $1400(19.8)$ & 3.1 & $1.241(0.894-1.722)$ & & 6.5 & $1.564(1.204-2.031)$ & \\
\hline Unknown & $1137(16.1)$ & & & & & & \\
\hline Ki67 & & & & 0.553 & & & 0.082 \\
\hline$\geq 14 \%$ & $724(10.2)$ & 4.8 & 1.000 & & 7.5 & 1.000 & \\
\hline$<14 \%$ & $712(10.1)$ & 6.1 & $1.143(0.734-1.780)$ & & 10.2 & $1.378(0.958-1.981)$ & \\
\hline Unknown & $5637(79.7)$ & & & & & & \\
\hline
\end{tabular}


Table 1 Local and regional recurrence rates stratified by patient, tumor, and treatment characteristics (Continued)

\begin{tabular}{|c|c|c|c|c|c|c|c|}
\hline Characteristic & No. of Patients (\%) & 5-year LR (\%) & HR $(95 \% \mathrm{Cl})$ & $P$ & 5-year RR (\%) & HR $(95 \% \mathrm{Cl})$ & $P$ \\
\hline \multicolumn{8}{|l|}{ Molecular subtypes } \\
\hline Luminal A & $2397(33.9)$ & 1.3 & 1.000 & $<0.001$ & 2.5 & 1.000 & $<0.001$ \\
\hline Luminal B-Her2 negative & $573(8.1)$ & 2.6 & $2.132(1.303-3.486)$ & & 5.3 & $1.920(1.264-2.917)$ & \\
\hline Luminal B-Her2 positive & $810(11.5)$ & 3.0 & $1.689(1.032-2.765)$ & & 5.8 & $1.944(1.329-2.842)$ & \\
\hline Her2-enriched & $583(8.2)$ & 3.4 & $2.029(1.231-3.343)$ & & 7.5 & $2.322(1.564-3.447)$ & \\
\hline Triple-negative & $967(13.7)$ & 4.9 & $2.711(1.842-3.991)$ & & 7.3 & $2.279(1.626-3.194)$ & \\
\hline Unknown & $1743(24.6)$ & & & & & & \\
\hline Endocrine therapy ${ }^{\mathrm{b}}$ & & & & 0.075 & & & 0.782 \\
\hline Yes & $4400(84.4)$ & 1.4 & 1.000 & & 2.9 & 1.000 & \\
\hline No & $569(10.9)$ & 2.7 & $1.535(0.954-2.470)$ & & 2.7 & $0.931(0.563-1.541)$ & \\
\hline Unknown & $243(4.7)$ & & & & & & \\
\hline Chemotherapy & & & & $<0.001$ & & & $<0.001$ \\
\hline No & $1662(23.5)$ & 1.3 & 1.000 & & 1.9 & 1.000 & \\
\hline Yes & $5373(76.0)$ & 2.8 & $2.424(1.561-3.765)$ & & 5.2 & $2.979(1.993-4.451)$ & \\
\hline Unknown & $38(0.5)$ & & & & & & \\
\hline Anti-Her2 target therapy ${ }^{c}$ & & & & 0.079 & & & 0.415 \\
\hline Yes & $321(22.9)$ & 1.0 & 1.000 & & 6.1 & 1.000 & \\
\hline No & $1067(76.2)$ & 3.6 & $2.437(0.871-6.814)$ & & 6.7 & $1.271(0.713-2.264)$ & \\
\hline Unknown & $12(0.9)$ & & & & & & \\
\hline
\end{tabular}

Abbreviations: HER2 human epidermal growth factor receptor 2

a For patients who did not receive neoadjuvant chemotherapy, we used pathological stage because it is more accurate than clinical stage. For patients who received neoadjuvant chemotherapy, we used whichever stage was higher (clinical or pathological) to reflect the actual tumor burden

${ }^{b}$ Only hormone-receptor positive patients included

' Only Her2 positive patients included

\section{Discussion}

The present study reports the patterns of LRR in a large cohort of post-mastectomy breast cancer patients who received modern treatment regimens, and the findings show that the SCN and chest wall recurrence rates were similar in the patient cohort, irrespective of the disease stage or molecular subtype, but axillary or IMN

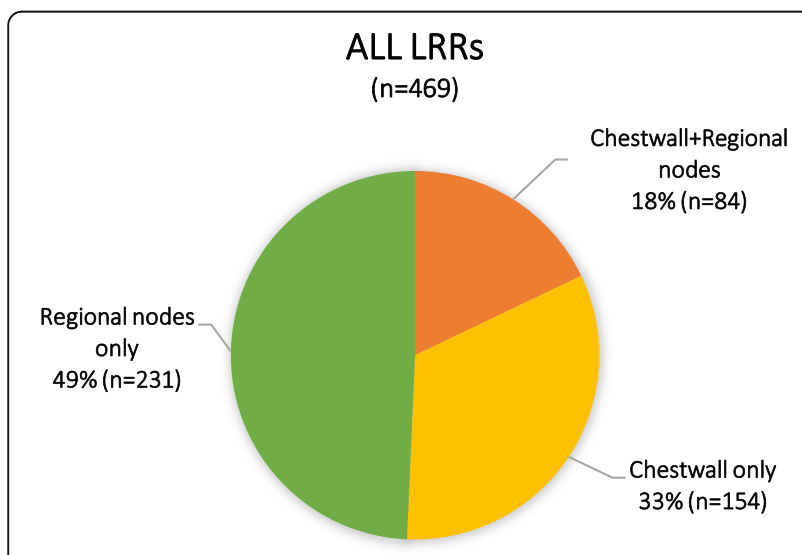

Fig. 2 Overall distribution of cumulative locoregional recurrences recurrence was relatively rare. This finding is slightly different from those of previous studies, as shown in Table $6[5,11-14,19-33]$, which show that the chest wall is the most common site of LRR. The decrease in chest wall recurrence is probably attributable to advances in surgical techniques that have made it possible to use thinner flaps for surgery. In the present cohort

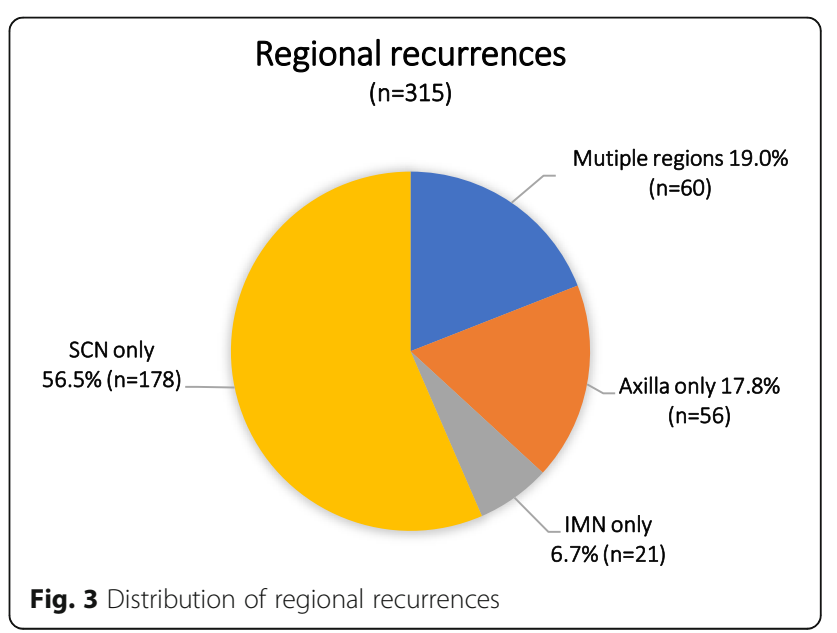


Table 2 The incidence and sites of LRR by risk group

\begin{tabular}{lllllll}
\hline Risk group & Total & LRR & CW & SCN & Axilla & IMN \\
& No. & No. (\%) & No. (\%) & No. (\%) & No. (\%) & No. (\%) \\
\hline Low Risk & 4606 & $170(3.7)$ & $92(54.1)$ & $68(40.0)$ & $27(15.9)$ & $27(15.9)$ \\
Intermediate Risk & 2042 & $185(9.1)$ & $83(44.9)$ & $110(59.5)$ & $37(20.0)$ & $20(10.8)$ \\
High Risk & 427 & $114(26.7)$ & $63(55.3)$ & $58(50.9)$ & $28(24.6)$ & $3(2.6)$ \\
Total cohort & 7073 & $469(6.6)$ & $238(50.7)$ & $236(50.3)$ & $92(19.6)$ & $50(10.7)$ \\
\hline
\end{tabular}

Abbreviations: CW chest wall; SCN supraclavicular/infraclavicular nodes; IMN internal mammary nodes; $L R R$ locoregional recurrence

analyzed, $95.2 \%$ of the patients had undergone axillary dissection, only $4.8 \%$ of the patients had undergone sentinel lymph node biopsy, although $65.1 \%$ of the patients had N0 disease. The usage of axillary dissection in the present study was similar to that of previous studies shown in Table 6. In addition, a randomized trial has established that similar low risk of axillary recurrence was found in N0 patients regardless of axillary procedures [34]. Therefore, axillary dissection may not be a factor in reducing the rate of recurrence recorded in axilla, with a consequent higher percentage of relapses recorded in the SCN. Whereas advances in radiology have resulted in an increase in the sensitivity of detection of SCN recurrence [35], and improvements in the effectiveness of systemic therapy have reduced the risk of LRR and might also change the LRR patterns, which might be the explanation for the high percentage of relapses recorded in the SCN.

The chest wall has been documented as the most common site of LRR so far, and it is generally included in the target volume when PMRT is administered. Regional nodal irradiation, especially SCN irradiation, is widely used for patients with heavy axillary nodal burden, for example, patients with four or more positive nodes. In this study, $26.7 \%$ of the patients in the high-risk group developed LRR: chest wall recurrence, $55.3 \%$; SCN recurrence, $50.9 \%$. The high recurrence rate in this group is consistent with the findings of several large-scale studies, which recommend both chest wall and SCN irradiation in these patients $[6,22,23,25,36]$.
It is unclear whether nodal irradiation can be omitted in those with a lower axillary nodal burden (e.g., those with less than four positive nodes). Among the intermediate-risk patients in this study, 9.1\% developed LRR: SCN recurrence in $59.5 \%$ of the cases and chest wall recurrence in $44.9 \%$ of the cases. However, in previous two small series focusing on $\mathrm{T} 1-2 \mathrm{~N} 1$ disease, the most common recurrence site was the chest wall (50-77\%), and SCN recurrence accounted for $23-35 \%$ of all recurrences [21, 27]. Similarly, Karlsson et al. reported that the 10-year recurrence rate for the chest wall, SCN, and axilla was 10.3, 2.6, and $4.8 \%$, respectively, for $\mathrm{N} 1$ patients who did not undergo PMRT [37]. In addition, some studies have suggested that regional nodal irradiation should be omitted in N1 patients, because of the low recurrence rate [38] or lack of positive outcome [39]. Further, according to the SUPREMO study [40], which is a randomized Phase III trial assessing the role of chest wall irradiation in women with intermediate-risk breast cancer following mastectomy, regional nodal irradiation was optional. In contrast, in the recent MA20 and EORTC 22922-10925 randomized controlled trials that included 85 and $43.1 \%$ patients with N1 disease, respectively, additional SCN and IMN irradiation significantly improved disease-free survival $[41,42]$. The findings of these two trials seem to corroborate our findings, which also indicated that $\mathrm{SCN}$ should be covered in PMRT for N1 breast cancer. This recommendation is important, as this recurrence

Table 3 The distribution of LRR by intrinsic molecular subtype

\begin{tabular}{lllllll}
\hline Molecular Subtypes & $\begin{array}{l}\text { Total } \\
\text { No. }\end{array}$ & LRR & CW & SCN & Axilla & IMN \\
& No. (\%) & No. (\%) & No. (\%) & No. (\%) & No. (\%) \\
\hline Luminal A & 2397 & $105(4.4)$ & $47(44.8)$ & $50(47.6)$ & $20(19.0)$ & $9(8.6)$ \\
Luminal B-Her2 negative & 573 & $42(7.3)$ & $24(57.1)$ & $23(54.8)$ & $11(26.2)$ & $7(16.7)$ \\
Luminal B-Her2 positive & 810 & $58(7.2)$ & $25(43.1)$ & $30(51.7)$ & $13(22.4)$ & $6(10.3)$ \\
Her2-enriched & 583 & $50(8.6)$ & $23(46.0)$ & $32(64.0)$ & $8(16.0)$ & $7(14.0)$ \\
Triple-negative & 967 & $100(10.3)$ & $58(58.0)$ & $50(50.0)$ & $21(21.0)$ & $12(12.0)$ \\
Total cohort & 5330 & $355(6.7)$ & $177(49.9)$ & $185(52.1)$ & $73(20.6)$ & $41(11.5)$ \\
\hline
\end{tabular}


Table 4 The distribution of LRR by targeted and endocrine therapy in Her2-positive and hormone receptor-positive patients

\begin{tabular}{|c|c|c|c|c|c|c|}
\hline Characteristic & Total No. & LRR & CW & SCN & Axilla & IMN \\
\hline & & No. (\%) & No. (\%) & No. (\%) & No. (\%) & No. (\%) \\
\hline Her2 positive & 1400 & $109(7.8)$ & $49(45.0)$ & $63(57.8)$ & $21(19.3)$ & $14(12.8)$ \\
\hline Targeted therapy & 321 & $16(5.0)$ & $4(25.0)$ & $9(56.3)$ & $2(12.5)$ & $4(25.0)$ \\
\hline No targeted therapy & 1067 & $92(8.6)$ & $44(47.8)$ & $53(57.6)$ & $19(20.7)$ & $10(10.9)$ \\
\hline Hormone receptor positive & 5212 & $281(5.4)$ & $139(49.5)$ & $134(47.7)$ & $57(20.3)$ & $25(8.9)$ \\
\hline Endocrine therapy & 4400 & $210(4.8)$ & $95(45.2)$ & $101(48.1)$ & $40(19.0)$ & $23(11.0)$ \\
\hline No endocrine therapy & 569 & $30(5.3)$ & $21(70.0)$ & $13(43.3)$ & $9(30.0)$ & $2(6.7)$ \\
\hline
\end{tabular}

Table 5 Multivariate analysis of prognostic factors for local recurrence and regional recurrence

\begin{tabular}{|c|c|c|c|c|}
\hline \multirow[t]{2}{*}{ Variable } & \multicolumn{2}{|l|}{ LR } & \multicolumn{2}{|l|}{$\mathrm{RR}$} \\
\hline & HR (95\% Cl) & $\mathrm{P}$ & $\mathrm{HR}(95 \% \mathrm{Cl})$ & $\mathrm{P}$ \\
\hline Age, y & & 0.001 & & $<0.001$ \\
\hline$>45$ & 1.000 & & 1.000 & \\
\hline$\leq 45$ & $1.688(1.232-2.313)$ & & $2.061(1.560-2.723)$ & \\
\hline Tumor location & & $<0.001$ & & 0.001 \\
\hline Other quadrants & 1.000 & & 1.000 & \\
\hline Inner quadrant & $2.059(1.488-2.851)$ & & $1.684(1.251-2.268)$ & \\
\hline No. of axillary nodes dissected & & 0.428 & & 0.299 \\
\hline$\geq 10$ & 1.000 & & 1.000 & \\
\hline$<10$ & $1.337(0.652-2.741)$ & & $1.407(0.738-2.682)$ & \\
\hline T Stage & & $<0.001$ & & $<0.001$ \\
\hline $\mathrm{T} 1-2$ & 1.000 & & 1.000 & \\
\hline T3-4 & $7.381(4.097-13.296)$ & & $3.585(2.025-6.349)$ & \\
\hline N Stage & & $<0.001$ & & $<0.001$ \\
\hline NO & 1.000 & & 1.000 & \\
\hline N1 & $2.070(1.455-2.944)$ & & $3.215(2.321-4.453)$ & \\
\hline N2 & $2.716(1.241-5.947)$ & & 5.169 (2.752-9.709) & \\
\hline N3 & $9.871(5.223-18.656)$ & & $12.636(7.215-22.131)$ & \\
\hline Tumor grade & & 0.144 & & 0.110 \\
\hline 1 & 1.000 & & 1.000 & \\
\hline 2 & $3.719(0.913-15.147)$ & & $1.433(0.626-3.282)$ & \\
\hline 3 & $4.109(0.992-17.010)$ & & $1.881(0.807-4.384)$ & \\
\hline Hormone receptor status & & 0.002 & & 0.007 \\
\hline Positive & 1.000 & & 1.000 & \\
\hline Negative & $1.741(1.236-2.454)$ & & $1.536(1.123-2.102)$ & \\
\hline Her2 status & & & & 0.535 \\
\hline Negative & & & 1.000 & \\
\hline Positive & & & $1.105(0.806-1.515)$ & \\
\hline Chemotherapy & & 0.256 & & 0.247 \\
\hline No & 1.000 & & 1.000 & \\
\hline Yes & $1.374(0.794-2.380)$ & & $1.371(0.804-2.337)$ & \\
\hline
\end{tabular}




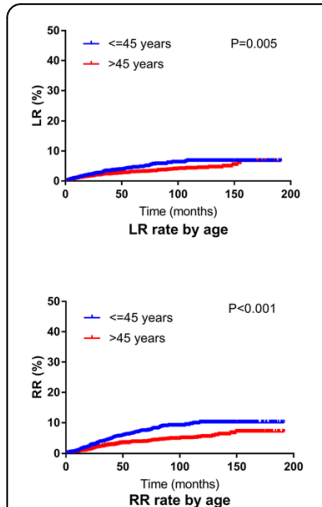

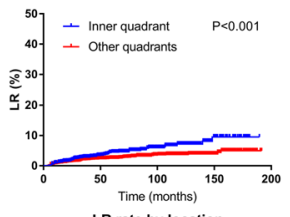

LR rate by location

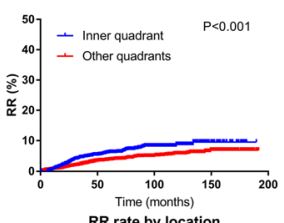

RR rate by location
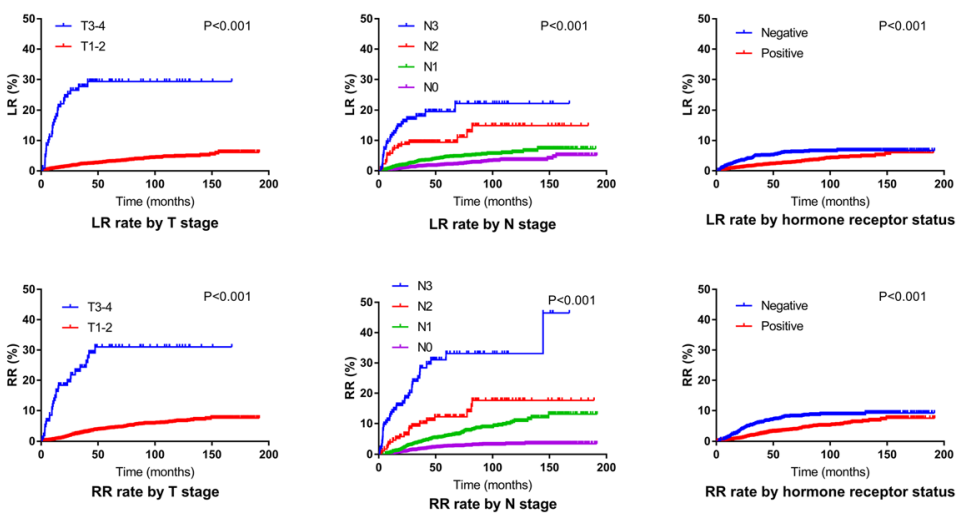

Fig. 4 Local and regional recurrence curves according to different prognostic factors for the entire cohort pattern is representative of the more recent trends in LRR in post-mastectomy breast cancer patients.

Among the low-risk patients (pT1-2 N0 disease) in this study, 3.7\% developed LRR: $54.1 \%$ in the chest wall and $40.0 \%$ in the SCN. A recent study revealed a similar distribution of LRR: $53.8 \%$ chest wall recurrence and $48.7 \%$ SCN recurrence [14]. In contrast, some previous studies have shown that chest wall recurrence accounted for $68.5-75.8 \%$, while SCN recurrence only accounted for $9.1-19.1 \%$ of all recurrences [11-13]. Further, in the study by Yildirim, all 14 LRRs were located in the chest wall [43]. Thus, recent findings have shown that the SCN might also be an important region to be covered in PMRT, and therefore, the traditional notion that the chest wall is the only site to be irradiated in pT1-2 N0 disease is being challenged.

It has been reported that the Her2-enriched and TN subtypes of breast cancer are the most susceptible to locoregional failure $[44,45]$. Further, patients with the $\mathrm{TN}$ subtype had a significantly higher risk of $\mathrm{RR}$ than those with the other subtypes, while no significant difference was found in the risk of LR [46-48]. Additionally, a German study showed that for all first local, regional, bone, and visceral recurrences of the Luminal A, Luminal B-Her2 negative, Luminal BHer2 positive, Her2-enriched, and TN subtypes, LR accounted for $13.8,20.0,1.1,6.4$, and $36.0 \%$ recurrences, respectively, while RR accounted for 21.1, 2.7, 9.5, 12.9, and 6.0\% recurrences, respectively [46]. The luminal subtypes were associated with bone recurrence, while the Her2-enriched and TN subtypes were associated with visceral recurrence [49]. Previous investigations generally did not distinguish between patients who underwent breast-conserving surgery and mastectomy, or between patients who did and did not undergo radiotherapy, and none of them specifically focused on LRR patterns based on molecular subtypes. This study tries to answer these questions: we found that the distribution of LRR sites was similar among the five molecular subtypes (the chest wall and SCN were the most common sites). Further, even in Her2-positive patients and hormone receptorpositive patients, the most common LRR sites were the chest wall and $\mathrm{SCN}$, irrespective of whether they underwent anti-HER2-targeted therapy (in the former group) or endocrine therapy (in the latter group). This finding indicates that PMRT must also cover $\mathrm{SCN}$, irrespective of the molecular subtype.

In this study, we found that the prognostic factors for LR and RR were similar; this means that it is necessary to cover both local and regional areas when administering PMRT. Very few studies have examined LR and RR separately. Several studies showed that age [30], $\mathrm{T}$ stage [25, 26], pathological type (ductal carcinoma vs. others) [26], and fascia invasion [26] was only associated with LR, and the number of positive nodes (3 vs. 1-2) [30], tumor grade [25], resection of only a few nodes $(<8)$ [25], and extracapsular invasion [25] was only associated with RR or axillary recurrence. These factors are mostly similar to those identified as being associated with both LR and RR in the present study; therefore, they should be considered when trying to predict locoregional failure in this group of patients.

The limitations of this study should be acknowledged. The main limitation is its retrospective design. Further, only half of the LRRs were confirmed by pathological examination. Despite these limitations, the sample was quite large, and to the best of our knowledge it is the first one to compare the LRR patterns among different disease stages and different molecular subtypes. In this study, we investigated the prognostic factors of LR and RR separately to find if regional nodal irradiation can be individualized, the results demonstrated the necessity to cover both local and regional area when PMRT was indicated. 


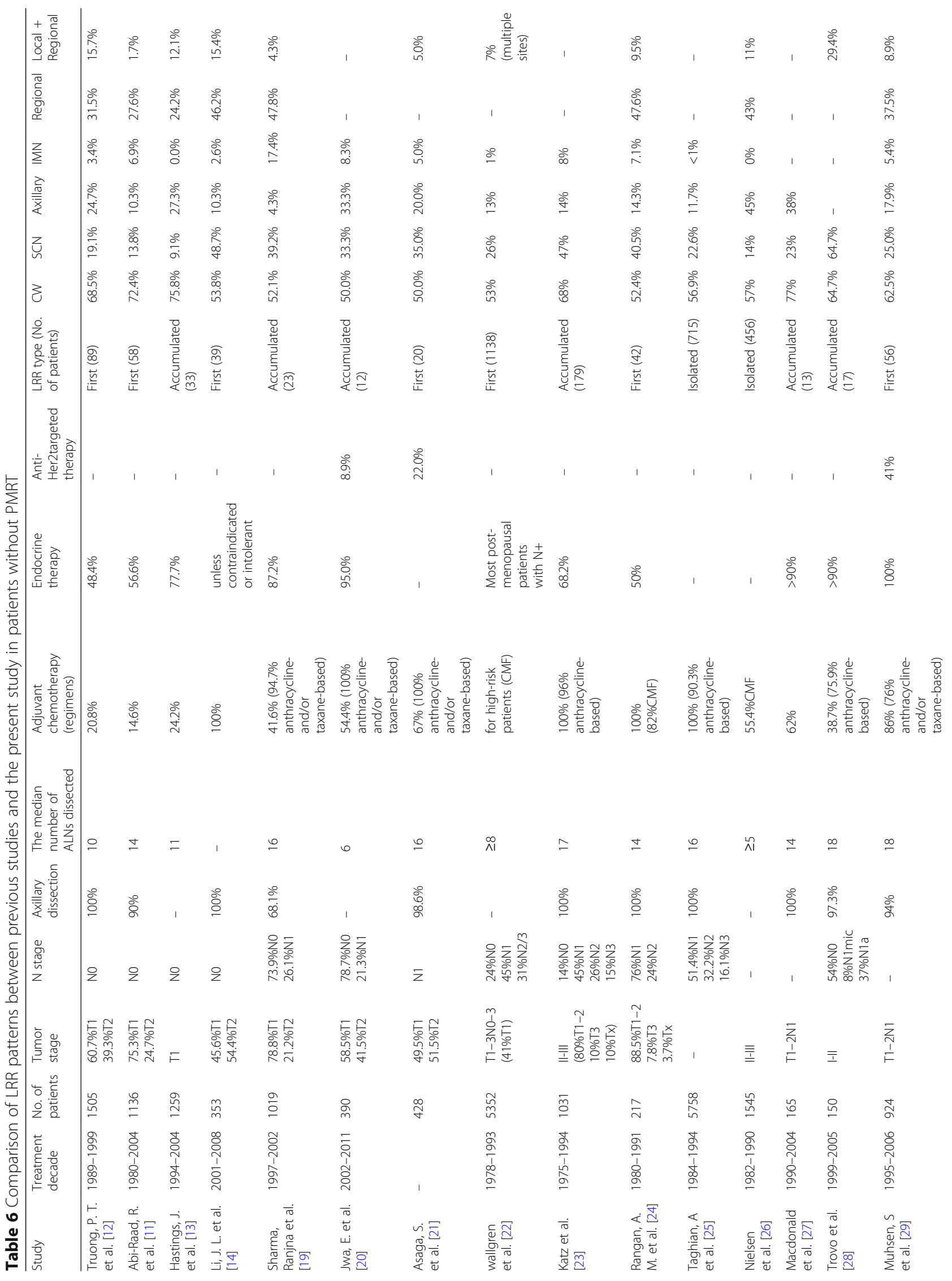




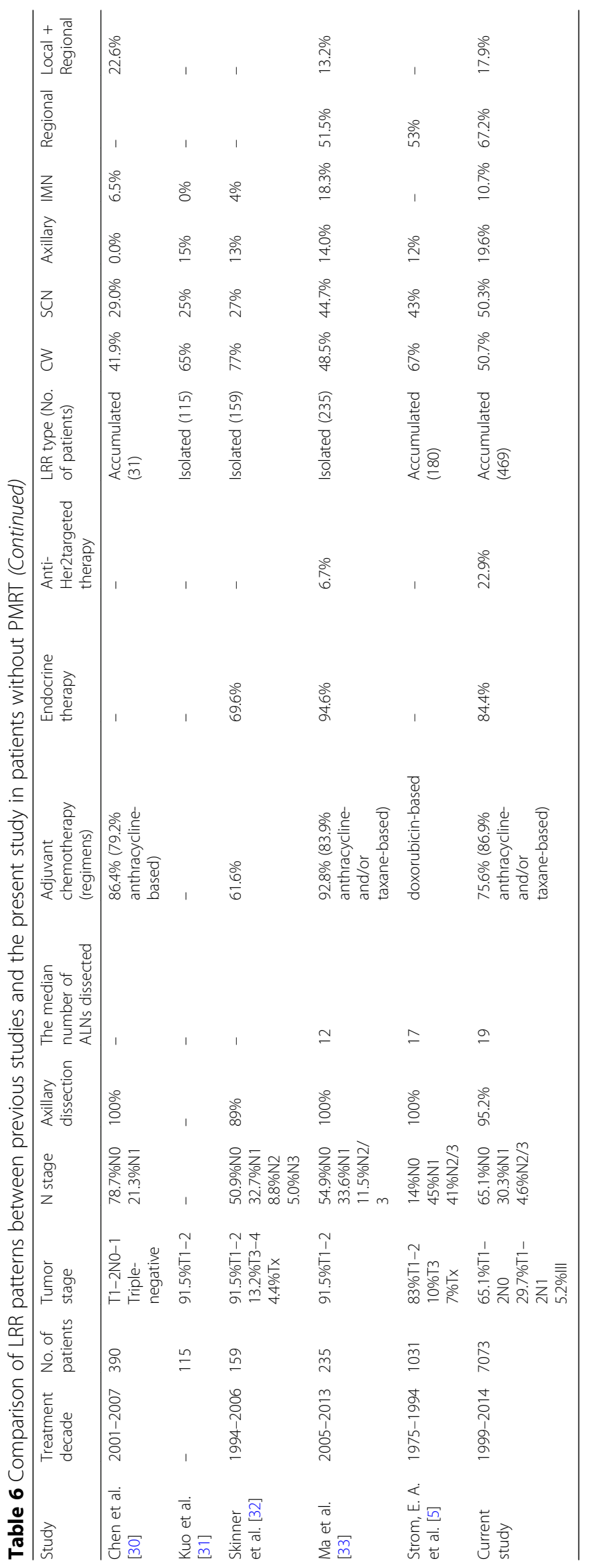




\section{Conclusion}

In summary, the findings of this study clearly show that the $\mathrm{SCN}$ region is as common as the chest wall as a recurrence site, irrespective of tumor stage or molecular subtype. Based on the findings, we recommended that SCN also be covered in all patients who undergo PMRT. Further, the relatively low incidence of axillary and IMN recurrence does not justify the routine application of radiotherapy to these regions.

\section{Supplementary information}

Supplementary information accompanies this paper at https://doi.org/10. 1186/s13014-020-01637-w.

Additional file 1: Table S1. The univariate and multivariate analysis of prognostic factors for SCN recurrence

\section{Abbreviations}

LRR: Locoregional recurrence; PMRT: Postmastectomy radiotherapy; LR: Local recurrence; RR: Regional recurrence; SCN: Supraclavicular/infraclavicular nodes; IMN: Internal mammary nodes; TNBC: Triple-negative breast cancer; IHC: Immunohistochemistry; FISH: Fluorescence in situ hybridization; CW: Chest wall; DM: Distant metastasis

\section{Acknowledgements}

Not applicable

\section{Authors' contributions}

Xuran Zhao: Formal analysis, investigation, data collection, methodology, and writing original draft. Yu Tang: Formal analysis, investigation, data collection, methodology, and writing original draft. Shulian Wang: Formal analysis and data collection, validation, statistics guidance, and project administration, patients care, and writing original draft and editing. Yong Yang: Patients care, and review original draft and editing. Hui Fang: Patients care, and review original draft and editing. Jianyang Wang: Data collection and review original draft and editing. Hao Jing: Data collection, and review original draft and editing. Jianghu Zhang: Patients care, and review original draft and editing. Guangyi Sun: Data collection and review original draft and editing. Siye Chen: Data collection and review original draft and editing. Jing Jin: Patients care, and review original draft and editing. Yongwen Song: Patients care, and review original draft and editing. Yueping Liu: Patients care, and review original draft and editing. Bo Chen: Patients care, and review original draft and editing. Shunan Qi: Patients care, and review original draft and editing. Ning Li: Patients care, and review original draft and editing. Yuan Tang: Patients care, and review original draft and editing. Ningning Lu: Patients care, and review original draft and editing. Hua Ren: Patients care, and review original draft and editing. Yexiong Li: Formal analysis, validation, statistics guidance, and project administration, patients care, and writing original draft and editing. All authors read and approved the final manuscript.

\section{Funding}

This study was supported by grants from the National Key Projects of Research and Development of China (2016YFC0904600); the Capital Characteristic Clinic Project (Z171100001017116) and the National Natural Science Foundation of China (81972860).

\section{Availability of data and materials}

The datasets used during the current study are available from the corresponding author on reasonable request.

Ethics approval and consent to participate

This study was approved by our Institutional Review Board (approval number 15-057/984).

\section{Consent for publication}

Not applicable.

\section{Competing interests}

The authors declare that they have no completing interests.

Received: 9 February 2020 Accepted: 6 August 2020

Published online: 04 September 2020

\section{References}

1. Clarke M, Collins R, Darby S, et al. Effects of radiotherapy and of differences in the extent of surgery for early breast cancer on local recurrence and 15-year survival: an overview of the randomised trials. Lancet. 2005:366:2087-106.

2. Ragaz J, Olivotto IA, Spinelli JJ, et al. Locoregional radiation therapy in patients with high-risk breast cancer receiving adjuvant chemotherapy: 20year results of the British Columbia randomized trial. J Natl Cancer Inst. 2005;97:116-26.

3. Overgaard M, Hansen PS, Overgaard J, et al. Postoperative radiotherapy in high-risk premenopausal women with breast cancer who receive adjuvant chemotherapy. Danish breast Cancer cooperative group 82b trial. N Engl J Med. 1997:337:949-55

4. Overgaard M, Jensen MB, Overgaard J, et al. Postoperative radiotherapy in high-risk postmenopausal breast-cancer patients given adjuvant tamoxifen: Danish breast Cancer cooperative group DBCG 82C randomised trial. Lancet. 1999;353:1641-8.

5. Strom EA, Woodward WA, Katz A, et al. Clinical investigation: regional noda failure patterns in breast cancer patients treated with mastectomy without radiotherapy. Int J Radiat Oncol Biol Phys. 2005;63:1508-13.

6. Recht A, Gray R, Davidson NE, et al. Locoregional failure 10 years after mastectomy and adjuvant chemotherapy with or without tamoxifen without irradiation: experience of the eastern cooperative oncology group. J Clin Oncol. 1999;17:1689-700.

7. Recht A, Pierce SM, Abner A, et al. Regional nodal failure after conservative surgery and radiotherapy for early-stage breast carcinoma. J Clin Oncol. 1991:9:988-96.

8. Vicini FA, Horwitz EM, Lacerna MD, et al. The role of regional nodal irradiation in the management of patients with early-stage breast cancer treated with breast-conserving therapy. Int J Radiat Oncol Biol Phys. 1997; 39:1069-76.

9. Yates $L$, Kirby $A$, Crichton $S$, et al. Risk factors for regional nodal relapse in breast cancer patients with one to three positive axillary nodes. Int J Radiat Oncol Biol Phys. 2012;82:2093-103.

10. Barrientos R, Samtani S, Frelinghuysen M, Sotomayor C, Gormaz JG, Burotto M. Clinical decision making in postmastectomy radiotherapy in node negative breast cancer. Ecancermedicalscience. 2018;12:874.

11. Abi-Raad R, Boutrus R, Wang R, et al. Patterns and risk factors of locoregional recurrence in T1-T2 node negative breast cancer patients treated with mastectomy: implications for postmastectomy radiotherapy. Int J Radiat Oncol Biol Phys. 2011;81:e151-7.

12. Truong PT, Lesperance M, Culhaci A, Kader HA, Speers $\mathrm{CH}$, Olivotto IA. Patient subsets with T1-T2, node-negative breast cancer at high locoregional recurrence risk after mastectomy. Int J Radiat Oncol Biol Phys. 2005;62:175-82.

13. Hastings J, Iganej S, Huang C, Huang R, Slezak J. Risk factors for locoregional recurrence after mastectomy in stage T1 N0 breast cancer. Am J Clin Oncol. 2014:37:486-91.

14. Li JL, Lin $X Y$, Zhuang $L$, et al. Establishment of a risk scoring system for predicting locoregional recurrence in T1 to T2 node-negative breast cancer patients treated with mastectomy: Implications for postoperative radiotherapy. Medicine (Baltimore). 2017;96:e7343.

15. Levine MN, Bramwell VH, Pritchard Kl, et al. Randomized trial of intensive cyclophosphamide, epirubicin, and fluorouracil chemotherapy compared with cyclophosphamide, methotrexate, and fluorouracil in premenopausal women with node-positive breast cancer. National Cancer Institute of Canada clinical trials group. J Clin Oncol. 1998;16:2651-8.

16. Abe O, Abe R, Enomoto K, et al. Effects of chemotherapy and hormonal therapy for early breast cancer on recurrence and 15-year survival: an overview of the randomised trials. Lancet. 2005;365:1687-717.

17. Henderson IC, Berry DA, Demetri GD, et al. Improved outcomes from adding sequential paclitaxel but not from escalating doxorubicin dose in an adjuvant chemotherapy regimen for patients with node-positive primary breast cancer. J Clin Oncol. 2003;21:976-83. 
18. Goldhirsch A, Winer EP, Coates AS, et al. Personalizing the treatment of women with early breast cancer: highlights of the St Gallen international expert consensus on the primary therapy of early breast Cancer 2013. Ann Oncol. 2013;24:2206-23.

19. Sharma R, Bedrosian I, Lucci A, et al. Present-day Locoregional control in patients with $\mathrm{T} 1$ or $\mathrm{T} 2$ breast Cancer with 0 and 1 to 3 positive lymph nodes after mastectomy without radiotherapy. Ann Surg Oncol. 2010;17: 2899-908.

20. Jwa $\mathrm{E}$, Shin $\mathrm{KH}$, Lim HW, et al. Identification of risk factors for Locoregional recurrence in breast Cancer patients with nodal stage N0 and N1: who could benefit from post-mastectomy radiotherapy? PLoS One. 2015;10: e0145463.

21. Asaga S, Kinoshita T, Shiino S, Jimbo K, Takayama S. Prognostic factors for breast cancer patients with T1-2 tumor and 1-3 positive axillary nodes treated using total mastectomy without radiotherapy. Breast J. 2019;25:26-33.

22. Wallgren A, Bonetti M, Gelber RD, et al. Risk factors for locoregional recurrence among breast cancer patients: results from international breast Cancer study group trials I through VII. J Clin Oncol. 2003;21:1205-13.

23. Katz A, Strom EA, Buchholz TA, et al. Locoregional recurrence patterns after mastectomy and doxorubicin-based chemotherapy: implications for postoperative irradiation. J Clin Oncol. 2000;18:2817-27.

24. Rangan AM, Ahern V, Yip D, Boyages J. Local recurrence after mastectomy and adjuvant CMF: implications for adjuvant radiation therapy. Aust N Z J Surg. 2000;70:649-55.

25. Taghian A, Jeong JH, Mamounas $\mathrm{E}$, et al. Patterns of locoregional failure in patients with operable breast cancer treated by mastectomy and adjuvant chemotherapy with or without tamoxifen and without radiotherapy: results from five National Surgical Adjuvant Breast and bowel project randomized clinical trials. J Clin Oncol. 2004;22:4247-54.

26. Nielsen HM, Overgaard M, Grau C, Jensen AR, Overgaard J. Loco-regional recurrence after mastectomy in high-risk breast cancer--risk and prognosis. An analysis of patients from the DBCG $82 \mathrm{~b} \& \mathrm{c}$ randomization trials. Radiother Oncol. 2006;79:147-55.

27. Macdonald SM, Abi-Raad RF, Alm El-Din MA, et al. Chest wall radiotherapy: middle ground for treatment of patients with one to three positive lymph nodes after mastectomy. Int J Radiat Oncol Biol Phys. 2009;75:1297-303.

28. Trovo M, Durofil E, Polesel J, et al. Locoregional failure in early-stage breast Cancer patients treated with radical mastectomy and adjuvant systemic therapy: which patients benefit from Postmastectomy irradiation? Int J Radiat Oncol Biol Phys. 2012;83:e153-e7.

29. Muhsen S, Moo TA, Patil S, et al. Most breast Cancer patients with T1-2 Tumors and one to three positive lymph nodes do not need Postmastectomy radiotherapy. Ann Surg Oncol. 2018;25:1912-20.

30. Chen X, Yu X, Chen J, et al. Analysis in early stage triple-negative breast cancer treated with mastectomy without adjuvant radiotherapy: patterns of failure and prognostic factors. Cancer. 2013;119:2366-74.

31. Kuo SH, Huang CS, Kuo WH, Cheng AL, Chang KJ, Chia-Hsien CJ. Comprehensive locoregional treatment and systemic therapy for postmastectomy isolated locoregional recurrence. Int J Radiat Oncol Biol Phys. 2008;72:1456-64

32. Skinner HD, Strom EA, Motwani SB, et al. Radiation dose escalation for locoregional recurrence of breast cancer after mastectomy. Radiat Oncol. 2013:8:13.

33. Ma J, Jiang R, Fan $L$, et al. Isolated locoregional recurrence patterns of breast cancer after mastectomy and adjuvant systemic therapies in the contemporary era. Oncotarget. 2015;6:36860-9.

34. Krag DN, Anderson SJ, Julian TB, et al. Sentinel-lymph-node resection compared with conventional axillary-lymph-node dissection in clinically node-negative patients with breast cancer: overall survival findings from the NSABP B-32 randomised phase 3 trial. Lancet Oncol. 2010;11:927-33.

35. Lee JH, Kim J, Moon HJ, et al. Supraclavicular lymph nodes detected by $18 \mathrm{~F}$ FDG PET/CT in cancer patients: assessment with 18F-FDG PET/CT and sonography. AJR Am J Roentgenol. 2012;198:187-93.

36. Recht A, Edge SB, Solin $\amalg$, et al. Postmastectomy radiotherapy: clinical practice guidelines of the American Society of Clinical Oncology. J Clin Oncol. 2001;19:1539-69.

37. Karlsson P, Cole BF, Chua BH, et al. Patterns and risk factors for locoregional failures after mastectomy for breast cancer: an international breast Cancer study group report. Ann Oncol. 2012;23:2852-8.

38. Stranzl H, Peintinger F, Ofner P, Prettenhofer U, Mayer R, Hackl A. Regional nodal recurrence in the management of breast cancer patients with one to three positive axillary lymph nodes. Outcome of patients following tangential irradiation without a separate nodal field. Strahlenther Onkol. 2004;180:623-8.

39. Kim H, Park W, Yu Jl, et al. Prognostic impact of elective supraclavicular nodal irradiation for patients with $\mathrm{N} 1$ breast Cancer after lumpectomy and Anthracycline plus Taxane-based chemotherapy (KROG 1418): a Multicenter case-controlled study. Cancer Res Treat. 2017;49:970-80.

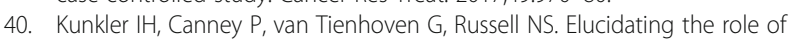
chest wall irradiation in 'intermediate-risk' breast cancer: the MRC/EORTC SUPREMO trial. Clin Oncol (R Coll Radiol). 2008;20:31-4.

41. Whelan TJ, Olivotto IA, Parulekar WR, et al. Regional nodal irradiation in early-stage breast Cancer. N Engl J Med. 2015;373:307-16.

42. Poortmans PM, Collette $\mathrm{S}$, Kirkove $\mathrm{C}$, et al. Internal mammary and medial supraclavicular irradiation in breast Cancer. N Engl J Med. 2015;373:317-27.

43. Yildirim E, Berberoglu U. Can a subgroup of node-negative breast carcinoma patients with T1-2 tumor who may benefit from postmastectomy radiotherapy be identified? Int J Radiat Oncol Biol Phys. 2007;68:1024-9.

44. Tsoutsou PG, Vozenin MC, Durham AD, Bourhis J. How could breast cancer molecular features contribute to locoregional treatment decision making? Crit Rev Oncol Hematol. 2017;110:43-8.

45. Lowery AJ, Kell MR, Glynn RW, Kerin MJ, Sweeney KJ. Locoregional recurrence after breast cancer surgery: a systematic review by receptor phenotype. Breast Cancer Res Treat. 2012;133:831-41.

46. Haffty $B G$, Yang $Q$, Reiss $M$, et al. Locoregional relapse and distant metastasis in conservatively managed triple negative early-stage breast cancer. J Clin Oncol. 2006;24:5652-7.

47. Sanpaolo P, Barbieri V, Genovesi D. Prognostic value of breast cancer subtypes on breast cancer specific survival, distant metastases and local relapse rates in conservatively managed early stage breast cancer: a retrospective clinical study. Eur J Surg Oncol. 2011;37:876-82.

48. Metzger-Filho $\mathrm{O}$, Sun Z, Viale G, et al. Patterns of recurrence and outcome according to breast cancer subtypes in lymph node-negative disease: results from international breast cancer study group trials VIII and IX. J Clin Oncol. 2013:31:3083-90.

49. Vasconcelos I, Hussainzada A, Berger S, et al. The St. Gallen surrogate classification for breast cancer subtypes successfully predicts tumor presenting features, nodal involvement, recurrence patterns and disease free survival. Breast. 2016;29:181-5.

\section{Publisher's Note}

Springer Nature remains neutral with regard to jurisdictional claims in published maps and institutional affiliations.

Ready to submit your research? Choose BMC and benefit from:

- fast, convenient online submission

- thorough peer review by experienced researchers in your field

- rapid publication on acceptance

- support for research data, including large and complex data types

- gold Open Access which fosters wider collaboration and increased citations

- maximum visibility for your research: over $100 \mathrm{M}$ website views per year

At BMC, research is always in progress.

Learn more biomedcentral.com/submissions 